Artículo

\title{
Desarrollo de plantas micropropagadas de Agave americana var. Oaxacensis durante su aclimatización en invernadero
}

\author{
Hermila Cruz García \\ Gisela Virginia Campos Ángeles ${ }^{\S}$ \\ José Raymundo Enríquez del Valle \\ Gerardo Rodríguez Ortiz \\ Vicente Arturo Velasco Velasco \\ Productividad en Agroecosistemas-TecNM-Instituto Tecnológico del Valle de Oaxaca. Ex-hacienda de \\ Nazareno, Xoxocotlán, Oaxaca. CP. 71233. Tel. 951 5170444. (mily_cg84@hotmail.com; \\ jenriquezdelvalle1959@gmail.com; geraro65@gmail.com; vicvel5@hotmail.com). \\ ${ }^{\S}$ Autor para correspondencia: giscampos@ hotmail.com.
}

\section{Resumen}

Agave americana var. Oaxacensis Gentry es una especie longeva que tarda aproximadamente 20 años en llegar a su etapa reproductiva. En Oaxaca, México, esta especie se usa como materia prima para elaborar la bebida destilada mezcal. Para incrementar el área de cultivo con esta especie, se propone propagarla in vitro, en complemento a los métodos de propagación convencionales. Las plantas micropropagadas deben pasar por un periodo de aclimatación, pero se desconocen las condiciones ambientales de adaptación, tales como el sustrato y abastecimiento de nutrientes las cuales afectan la sobrevivencia y magnitud de crecimiento de las plantas. El objetivo de la investigación fue evaluar las características morfológicas de plantas micropropagadas de $A$. americana var. Oaxacensis que se sometieron a prueba de diferentes mezclas de sustratos: turbaarena: $33.3 \%+66.6 \% \mathrm{v} / \mathrm{v}, 66.6 \%+33.3 \%$, v/v $75 \%+25 \% \mathrm{v} / \mathrm{v}$; así como, el abastecimiento de nutrientes mediante fertirriego con diluciones 0, 50 y $100 \%$ de la formulación de Steiner, durante 290 días de su aclimatización para lo que se utilizó un diseño completamente al azar con arreglo factorial, al término de este periodo, de un total de 135 plantas, el 100\% de éstas sobrevivió. Las plantas que alcanzaron mayor tamaño tuvieron las siguientes características: 5.5-5.6 hojas, la hoja mayor de 17.7 a $18.2 \mathrm{~cm}$ de longitud, 2.4 a $2.5 \mathrm{~cm}$ de ancho, el tallo de 2.4 a $2.5 \mathrm{~cm}$ de diámetro, y correspondieron a las establecidas en sustrato con $66.6 \%$ turba+ $33.3 \%$ arena v/v o $75 \%$ turba $+25 \%$ arena $\mathrm{v} / \mathrm{v}$, y que además se fertirrigaron. Las plantas fertirrigadas con solución nutritiva al $100 \%$ lograron tamaño mayor que las plantas irrigadas con solo agua.

Palabras clave: clorofila, estomas, fertirrigación.

Recibido: junio de 2019

Aceptado: agosto de 2019 


\section{Introducción}

La mayor parte de las especies de agave que se establecen en plantaciones comerciales, $A$. tequilana. A. sisalana, A. fourcroydes y A. angustifolia, se propagan convencionalmente por métodos asexuales, por ejemplo: hijuelos de rizoma o los que se originan de las hojas de las rosetas (hijuelos intrafoliares) que se desarrollan cuando se separan de la planta madre o ésta muere (Nobel, 1998; García-Mendoza, 2007), además existen los bulbilos que se originan a partir de yemas vegetativas en la inflorescencia, su desarrollo se induce cuando ocurre la abscisión o corte de los botones florales (Arizaga y Ezcurra, 1995).

Desde principios de la década de los 1980's en diversos países y particularmente en México se realizan investigaciones para la propagación in vitro de agaves (Arizaga y Ezcurra, 1995) por lo que se han podido describir las condiciones de manejo que se sugieren en cada etapa del proceso de micropropagación (Domínguez et al., 2008; Enríquez-del Valle, 2008), como una alternativa complementaria y no excluyente de los métodos convencionales. Actualmente se tienen reportes sobre la propagación in vitro de A. tequilana (Santacruz-Ruvalcaba et al., 2008), A. angustifolia (Enríquez-del Valle, 2008; Arzate-Fernández et al., 2016), A. inaequidens Koch (AureolesRodríguez et al., 2008), Agave fourcroydes Lem (Garriga et al., 2010), Agave cocui (González et al., 2012), Agave grijalvensis (Santíz et al., 2012), Agave sisalana (Carneiro-dos Santos et al., 2014). Sin embargo, hay otros agaves con valor biológico-social que han sido poco estudiados, como el Agave americana var. Oaxacensis Gentry, que es una especie endémica de México, que en Oaxaca es utilizada para la obtención de fibras duras (Palma-Cruz, 2000) y para la elaboración de la bebida destilada denominada mezcal (García-Mendoza, 2011). La producción de esta bebida usando como materia prima la especie mencionada, es limitada debido a que hay pocos ejemplares los cuales tardan hasta 20 años en llegar a la etapa de madurez para su cosecha.

Por lo que los agricultores han preferido propagar otras especies de ciclo reproductivo más corto, tal como el Agave angustifolia, que se cosecha entre los siete y nueve años de cultivo (CONABIO, 2006). En vista de lo anterior, para responder al interés por establecer plantaciones de esta especie, se sugiere aplicar la técnica de cultivo de tejidos vegetales o cultivo in vitro para su propagación en gran escala, pero también con fines de conservación, y aprovechamiento sustentable.

La técnica de cultivo de tejidos vegetales consiste en la producción intensiva de plantas en grandes cantidades, genéticamente homogéneas, en condiciones controladas de laboratorio y en espacios pequeños donde es posible manipular el material vegetal durante todo el año (George y Debergh, 2008). El proceso se realiza en secuencia de varias etapas: 1) selección del germoplasma o material vegetal; 2) establecimiento de cultivos asépticos; 3) multiplicación de propágulos; 4) enraizado de brotes en preparación para su trasplante a suelo; y 5) aclimatización de las plantas (George y Debergh, 2008; Enríquez-del Valle, 2008).

La etapa cuatro se considera de gran importancia porque es cuando las plántulas se transfieren de las condiciones in vitro a contenedores con sustratos y condiciones de invernadero, para promover que ocurran cambios graduales en su morfología y metabolismo, en el trascurso de 40 a 90 días, en preparación para su trasferencia posterior a vivero y campo. En el caso de los agaves se han desarrollado metodologías de micropropagación; sin embargo, a pesar de que se han establecido los procedimientos generales del cultivo de tejidos vegetales, es necesario determinar los 
requerimientos específicos de propagación in vitro en cada especie. Además, de evaluar el desempeño de las plantas micropropagadas en las etapas posteriores de aclimatación, invernadero y cultivo en campo (Enríquez-del Valle et al., 2016).

En algunos estudios sobre la aclimatización de plantas de agave micropropagadas se ha demostrado en A. tequilana (Crespo-González et al., 2013) y A. potatorum Zucc. (Enríquez-del Valle et al., 2016) que logran mayor crecimiento cuando reciben abastecimiento adecuado de nutrimentos, ya sea mediante soluciones nutritivas o incorporando fertilizantes minerales a los sustratos, comparadas con plantas que no reciben fertilización. Sin embrago, Para A. americana var. Oaxacensis no existen antecedentes al respecto, por lo que el objetivo del estudio fue evaluar el crecimiento y desarrollo de las plantas micropropagadas de Agave americana. var. Oaxacensis Gentry como respuesta a diferentes condiciones de sustrato y dosis de fertirriego durante su a climatización en invernadero.

\section{Materiales y métodos}

\section{Obtención de plantas}

La investigación es continuación del trabajo de Cruz-García et al. (2017), que se realizó en el laboratorio de cultivo de tejidos vegetales e invernaderos del Instituto Tecnológico del Valle de Oaxaca, ubicado en Nazareno, Xoxocotlán, Oaxaca. Las plantas de A. americana. var. Oaxacensis que se utilizaron, se obtuvieron mediante la formación de brotes a partir de tejido de tallo y su enraizado posterior in vitro; tenían en promedio 6.75 hojas, 5 raíces y su hoja más larga tenía 9.78 $\mathrm{cm}$ de longitud y $0.64 \mathrm{~cm}$ de ancho. Se trasplantaron a macetas de polietileno con capacidad de $171 \mathrm{~cm}^{3}$, que contenían diferentes sustratos.

Éstos consistieron en mezclas de turba y arena en diferente proporción (33.3-66.6\%, 66.6-33.3\%, $75-25 \%$ ) en relación a su volumen. Su aclimatización se realizó en una casa sombra tipo capilla, de $5.5 \mathrm{~m}$ de ancho, $12.1 \mathrm{~m}$ de longitud, $3.5 \mathrm{~m}$ de altura, con estructura metálica, techado de lámina acanalada traslúcida y cubiertas laterales de polietileno traslúcido, con radiación solar disminuida al $40 \%$, temperatura de 14 a $28{ }^{\circ} \mathrm{C}$, humedad relativa alta $80-90 \%$. Las plantas se mantuvieron durante 45 días en estas condiciones, recibiendo una vez por semana $10 \mathrm{~mL}$ de la solución universal Steiner (1984), diluida al 10\% de concentración. Posteriormente, a partir del día 46, las plantas se trasladaron a un segundo invernadero donde fueron expuestas a mayor radiación solar, temperatura entre 15 y $38{ }^{\circ} \mathrm{C}$, y humedad relativa entre 30 y 50\%, en estas condiciones estuvieron hasta el día 270, durante este tiempo la aportación de la solución nutritiva se aplicó a nivel de sustrato tres veces por semana, de acuerdo con los tratamientos establecidos.

\section{Diseño experimental y análisis de datos}

El experimento, se estableció de acuerdo con un diseño completamente al azar con arreglo factorial 3 x 3, en donde: 1) el factor sustrato se estableció en tres niveles: a) turba+arena en mezcla $33.3+66.6 \% \mathrm{v} / \mathrm{v}$, turba-arena en mezcla $66.6+33.3 \% \mathrm{v} / \mathrm{v}$, turba+arena en mezcla $75-25 \% \mathrm{v} / \mathrm{v}$ y, 2) el factor tipo de riego también se estableció en tres niveles (agua y solución nutritiva (SN) Steiner (1984), en dilución a 50\% y SN a 100\%). Por lo que resultaron nueve tratamientos. La SN (1984) a $100 \%$ contiene en $\mathrm{mg} \mathrm{L}^{-1}: 166.01 \mathrm{~N}, 31.35 \mathrm{P}, 277.38 \mathrm{~K}, 182.06 \mathrm{Ca}, 49.08 \mathrm{Mg}, 110.898 \mathrm{~S}, 1.33$ Fe, 0.201 Mn, 0.077 B, 0.019 Mo, 0.0375 Zn y 0.00065 Cu. 
La unidad experimental fue una planta y se tuvieron 15 repeticiones por tratamiento. El experimento concluyó a los 270 días de aclimatización y en ese momento se seleccionaron seis plantas por cada tratamiento a las que se le determinaron características morfológicas como: número de hojas (NH); longitud (LHM) y ancho $(\mathrm{AH})$ de la hoja mayor, peso fresco foliar (PFF) y peso seco foliar (PSF), volumen (VF) y área foliar (AF), suculencia foliar (SF), diámetro de tallo (DT), número de raíces principales (NRP), longitud de raíz (LR), volumen de raíz (VR), peso fresco de raíz (PFR), peso seco de raíz (PSR), índice estomático y contenido de clorofila. La suculencia foliar (SF), se determinó con la fórmula propuesta por Mantovani (1999), SF= (peso fresco máximo-peso seco $(\mathrm{g}))$ /área superficial $\left(\mathrm{cm}^{2}\right)$.

El contenido de clorofila se determinó en hojas adultas y jóvenes a través del método propuesto por Moran (1982), utilizando un medidor Minolta SPAD-502. Los valores del SPAD, se convirtieron a $\mu \mathrm{g} \mathrm{cm}^{-2}$ mediante la fórmula propuesta por Coste et al. (2010). Para la determinación de estomas, se utilizaron hojas de la parte media de la roseta, de ellas se eligieron tres secciones: basal, media y apical, tanto de la parte adaxial como abaxial, las muestras obtenidas se observaron en un microscopio compuesto marca Carl Zeiss, a 40x, posteriormente se contabilizaron el total de células y estomas.

Con los datos anteriores se calculó el índice estomático (IE) con la fórmula propuesta por Wilkinson (1979). Donde: IE= número de estomas por unidad de área/(número de estomas por unidad de área + número de células epidérmicas por unidad de área $) \times 100$. Los datos obtenidos de cada variable se sometieron a análisis de varianza y la prueba de Tukey para la comparación múltiple de medias con un nivel de significancia $\alpha=0.05$. Para la rutina de análisis se usó el paquete estadístico SAS ${ }^{\circledR}$ (The SAS System for Windows 9.0), se realizó el análisis de varianza correspondiente y la comparación múltiple de medias para establecer que tratamientos provocaron efectos significativos sobre las variables estudiadas.

\section{Resultados y discusión}

\section{Características de plantas micropropagadas de Agave americana var. Oaxacensis al finalizar su aclimatización}

Los análisis de varianza del efecto del sustrato y la fertilización sobre los indicadores del crecimiento y desarrollo de las plantas (Cuadros 1 y 2), muestran diferencias significativas en el efecto de los tratamientos. Mientras que la comparación múltiple de medias detectó que las diferencias $(p<0.01)$, provocadas por los niveles del factor sustrato se manifiestan sobre la mayoría de las variables, excepto para la suculencia foliar. Los niveles del factor tipo de riego tuvieron efectos significativos diferentes $(p<0.01)$ sobre la variable número de hojas $(\mathrm{NH})$, ancho de hoja $(\mathrm{AH})$, peso fresco foliar (PFF), volumen foliar (VF), área foliar (AF), diámetro de tallo (DT) y número de raíces principales (NRP). La interacción sustrato-tipo de riego mostró efectos significativos $(p<0.01)$ sobre NH, AHM, PFF, VF, AF y NRP. En un estudio anterior Cruz-García et al. (2017), reportaron que plantas de la misma especie, poseían hojas delgadas de consistencia herbácea al inicio de su aclimatización y que durante 240 días en esta etapa; ocurrió la senescencia gradual de las hojas provenientes del cultivo in vitro y que estas hojas fueron sustituidas por hojas nuevas que fueron más grandes, de 11.6 a $18.2 \mathrm{~cm}$ de longitud, 1.7 a $2.5 \mathrm{~cm}$ de ancho, engrosadas, y de consistencia herbácea pero más rígidas. 
También señalan que al término de esa etapa $100 \%$ de las plantas sobrevivió y que aunque tuvieron menos hojas que al inicio de la aclimatación, fueron más largas y anchas, aunque su tamaño estuvo en relación con la cantidad de nutrimentos que recibieron.

En este caso, cuando transcurrieron nueve meses las plantas más pequeñas fueron las que se establecieron en el sustrato $33.3 \%$ de turba $\mathrm{v} / \mathrm{v}+66.6 \%$ arena $\mathrm{v} / \mathrm{v}$, irrigadas con solo agua o con la $\mathrm{SN}-50 \%$. Conforme se incrementó la cantidad de turba en el sustrato hasta $66.6 \%$ y $75 \%$, las plantas alcanzaron tamaños mayores. Así también, las plantas fertirrigadas hasta SN-100\% tendieron a ser más grandes que las plantas irrigadas con solo agua.

Por lo que las plantas que se establecieron en los sustratos con $66 \%$ turba y $33.3 \%$ arena o $75 \%$ turba+25\% arena y que se fertirrigaron con la solución nutritiva a $100 \%$ de concentración de nutrimentos, desarrollaron 5.6 y 5.5 hojas, la hoja mayor fue de 17.7 y $18.2 \mathrm{~cm}$ de longitud, así como 2.5 y $2.4 \mathrm{~cm}$ de ancho, 34.9 y $31.8 \mathrm{~g}$ de peso fresco foliar, 36.1 y $34.5 \mathrm{~cm}^{3}$ de volumen foliar, 122.7 y $112 \mathrm{~cm}^{2}$ de área foliar, 4.06 y $3.61 \mathrm{~g}$ de peso seco foliar; el tallo de 2.5 y $2.4 \mathrm{~cm}$ de diámetro, 6.5 y 7.3 raíces, de 39.8 y $32.6 \mathrm{~cm}$ de longitud; la raíz de 12.5 y $10.8 \mathrm{~cm}^{3}$ de volumen; 10.7 y 9.9 $\mathrm{g}$ de peso fresco de raíz, 1.7 y $1.4 \mathrm{~g}$ de peso seco de raíz, magnitudes que fueron significativamente (Tukey, $p<0.05$ ) mayores a los valores registrados para las mismas variables en las plantas que se establecieron en sustrato con la mayor proporción de arena (33.3\% turba+ $66.6 \%$ arena) y que no fueron fertirrigadas (Cuadros 1 y 2).

Cuadro 1. Efecto del sustrato y fertilización sobre los indicadores de crecimiento y desarrollo de las hojas de plantas micropropagadas de $A$. americana que durante 270 días estuvieron en aclimatización en invernadero.

\begin{tabular}{cccccccccc}
\hline Sust & $\begin{array}{c}\text { Fert } \\
(\%)\end{array}$ & NH & $\begin{array}{c}\text { LHM } \\
(\mathrm{cm})\end{array}$ & $\begin{array}{c}\mathrm{AH} \\
(\mathrm{cm})\end{array}$ & PFF $(\mathrm{g})$ & $\begin{array}{c}\mathrm{VF} \\
\left(\mathrm{cm}^{3}\right)\end{array}$ & AF $\left(\mathrm{cm}^{2}\right)$ & PSF $(\mathrm{g})$ & SF \\
\hline $33.3 \% \mathrm{~T}:$ & 0 & $3.3 \mathrm{e}$ & $11.8 \mathrm{c}$ & $1.7 \mathrm{c}$ & $10.5 \mathrm{~b}$ & $11.5 \mathrm{~b}$ & $38.6 \mathrm{~d}$ & $1.15 \mathrm{c}$ & $0.3 \mathrm{a}$ \\
$66.6 \% \mathrm{~A}$ & 50 & $3.5 \mathrm{ed}$ & $12.7 \mathrm{bc}$ & $1.7 \mathrm{c}$ & $11.9 \mathrm{~b}$ & $13.1 \mathrm{~b}$ & $48.5 \mathrm{~cd}$ & $1.54 \mathrm{c}$ & $0.26 \mathrm{a}$ \\
& 100 & $3.8 \mathrm{cde}$ & $11.6 \mathrm{c}$ & $1.7 \mathrm{c}$ & $12.8 \mathrm{~b}$ & $14.1 \mathrm{~b}$ & $50.5 \mathrm{~cd}$ & $1.56 \mathrm{c}$ & $0.28 \mathrm{a}$ \\
$66.6 \% \mathrm{~T}:$ & 0 & $4.8 \mathrm{abc}$ & $16.6 \mathrm{ab}$ & $2.4 \mathrm{ab}$ & $22.1 \mathrm{ab}$ & $23.3 \mathrm{ab}$ & $84 \mathrm{abc}$ & $2.2 \mathrm{bc}$ & $0.28 \mathrm{a}$ \\
$33.3 \% \mathrm{~A}$ & 50 & $4.1 \mathrm{cde}$ & $15.3 \mathrm{abc}$ & $2 \mathrm{bc}$ & $22.3 \mathrm{ab}$ & $22.6 \mathrm{ab}$ & $73.2 \mathrm{bcd}$ & $1.94 \mathrm{c}$ & $0.3 \mathrm{a}$ \\
& 100 & $5.6 \mathrm{a}$ & $17.7 \mathrm{a}$ & $2.5 \mathrm{a}$ & $34.9 \mathrm{a}$ & $36.1 \mathrm{a}$ & $122.7 \mathrm{a}$ & $4.06 \mathrm{a}$ & $0.3 \mathrm{a}$ \\
$75 \% \mathrm{~T}:$ & 0 & $4.5 \mathrm{bcd}$ & $15 \mathrm{abc}$ & $1.9 \mathrm{bc}$ & $17 \mathrm{~b}$ & $18 \mathrm{~b}$ & $63.6 \mathrm{~cd}$ & $1.93 \mathrm{c}$ & $0.28 \mathrm{a}$ \\
$25 \% \mathrm{~A}$ & 50 & $4.5 \mathrm{bcd}$ & $15.1 \mathrm{abc}$ & $1.9 \mathrm{bc}$ & $17 \mathrm{~b}$ & $18.1 \mathrm{~b}$ & $62.9 \mathrm{~cd}$ & $2.07 \mathrm{bc}$ & $0.29 \mathrm{a}$ \\
& 100 & $5.5 \mathrm{ab}$ & $18.2 \mathrm{a}$ & $2.4 \mathrm{a}$ & $31.8 \mathrm{a}$ & $34.5 \mathrm{a}$ & $112 \mathrm{ab}$ & $3.61 \mathrm{ab}$ & $0.31 \mathrm{a}$ \\
Sust & & $* *$ & $* *$ & $* *$ & $* *$ & $* *$ & $* *$ & $* *$ & $\mathrm{~ns}$ \\
Fert & $* *$ & $\mathrm{~ns}$ & $* *$ & $* *$ & $* *$ & $* *$ & $* *$ & $\mathrm{~ns}$ \\
Sust $*$ Fert & & $* *$ & $\mathrm{~ns}$ & $* *$ & $*$ & $*$ & $* *$ & $\mathrm{~ns}$ & $\mathrm{~ns}$ \\
\hline
\end{tabular}

Sus=sustrato; Fert= fertilización; $33.3 \% \mathrm{~T}: 66.6 \% \mathrm{~A}=$ una porción de turba combinada con dos de arena; $66.6 \% \mathrm{~T}=$ $33.3 \% \mathrm{~A}=$ dos porciones de turba combinada con una de arena $75 \% \mathrm{~T}: 25 \% \mathrm{~A}=$ tres porciones de turba combinada con una de arena; $\mathrm{NH}=$ número de hojas; $\mathrm{LHM}=$ longitud de hoja mayor; $\mathrm{AH}=$ ancho de hoja; $\mathrm{PFF}=$ peso fresco foliar; $\mathrm{VF}=$ volumen foliar; $\mathrm{AF}=$ área foliar; $\mathrm{PSF}=$ peso seco foliar; $\mathrm{SF}=$ suculencia foliar; ${ }^{* *}=$ valor de $\mathrm{F}$ significativo $(p \leq$ $0.01) ;{ }^{*}=$ valor de $\mathrm{F}$ significativo $(p \leq 0.05)$; ns $=$ valor de $\mathrm{F}$ no significativo $(p \geq 0.05)$. 
Cuadro 2. Efecto del sustrato y fertilización sobre los indicadores de crecimiento y desarrollo de los tallos y raíces de plantas micropropagadas de $A$. americana que durante 270 días estuvieron en aclimatización en invernadero.

\begin{tabular}{|c|c|c|c|c|c|c|c|}
\hline Sust & Fert $(\%)$ & DT (cm) & NRP & $\mathrm{LR}(\mathrm{cm})$ & $\operatorname{VR}\left(\mathrm{cm}^{3}\right)$ & PFR (g) & PSR (g) \\
\hline $33.3 \% \mathrm{~T}:$ & 0 & $1.7 \mathrm{c}$ & $2.8 \mathrm{e}$ & $18 \mathrm{c}$ & $3 \mathrm{~d}$ & $2.3 \mathrm{c}$ & $0.5 \mathrm{c}$ \\
\hline \multirow[t]{2}{*}{$66.6 \% \mathrm{~A}$} & 50 & $1.7 \mathrm{c}$ & $3.1 \mathrm{de}$ & $19.1 \mathrm{c}$ & $3.6 \mathrm{~d}$ & $3.1 \mathrm{bc}$ & $0.6 \mathrm{c}$ \\
\hline & 100 & $1.8 \mathrm{c}$ & $3.1 \mathrm{de}$ & $16.5 \mathrm{c}$ & $4.1 \mathrm{~cd}$ & $3.4 \mathrm{bc}$ & $0.6 \mathrm{c}$ \\
\hline $66.6 \% \mathrm{~T}:$ & 0 & $1.9 \mathrm{bc}$ & $5.1 \mathrm{bcd}$ & $32.5 \mathrm{ab}$ & $10.8 \mathrm{ab}$ & $9.4 \mathrm{a}$ & $1.3 \mathrm{ab}$ \\
\hline \multirow[t]{2}{*}{$33.3 \% \mathrm{~A}$} & 50 & $1.9 \mathrm{bc}$ & $5.3 \mathrm{abc}$ & $31.6 \mathrm{ab}$ & $9.1 \mathrm{abc}$ & $8.1 \mathrm{ab}$ & $1.1 \mathrm{abc}$ \\
\hline & 100 & $2.5 \mathrm{a}$ & $6.5 \mathrm{ab}$ & $39.8 \mathrm{a}$ & $12.5 \mathrm{a}$ & $10.7 \mathrm{a}$ & $1.7 \mathrm{a}$ \\
\hline 75\% T: & 0 & $1.9 \mathrm{bc}$ & 4.1 cde & $27.5 \mathrm{bc}$ & 7 bcd & $6.4 \mathrm{abc}$ & $0.9 \mathrm{bc}$ \\
\hline \multirow[t]{2}{*}{$25 \% \mathrm{~A}$} & 50 & $1.9 \mathrm{bc}$ & $4.6 \mathrm{cde}$ & $25.5 \mathrm{bc}$ & $6.6 \mathrm{bcd}$ & $6.1 \mathrm{abc}$ & $0.8 \mathrm{bc}$ \\
\hline & 100 & $2.4 \mathrm{ab}$ & $7.3 \mathrm{a}$ & $32.6 \mathrm{ab}$ & $10.8 \mathrm{ab}$ & $9.9 \mathrm{a}$ & $1.4 \mathrm{ab}$ \\
\hline Sust & & $* *$ & $* *$ & $* *$ & $* *$ & $* *$ & $* *$ \\
\hline Fert & & ** & $* *$ & ns & ns & ns & $* *$ \\
\hline Sust*Fert & & $\mathrm{ns}$ & ** & $\mathrm{ns}$ & $\mathrm{ns}$ & ns & $\mathrm{ns}$ \\
\hline
\end{tabular}

Sus = sustrato; Fert $=$ fertilización; $33.3 \% \mathrm{~T}: 66.6 \% \mathrm{~A}=$ una porción de turba combinada con dos de arena; $66.6 \% \mathrm{~T}$ : $33.3 \% \mathrm{~A}=$ dos porciones de turba combinada con una de arena; $75 \% \mathrm{~T}: 25 \% \mathrm{~A}=$ tres porciones de turba combinada con una de arena; $\mathrm{DT}=$ diámetro del tallo; $\mathrm{NRP}=$ número de raíces principales; $\mathrm{LR}=$ longitud de raíz principal; $\mathrm{VR}=$ volumen de raíz; $\mathrm{PFR}=$ peso fresco de raíz; PSR= peso seco de raíz; ${ }^{* *}=$ valor de $\mathrm{F}$ significativo $(p \leq 0.01) ;{ }^{*}=$ valor de F significativo $(p \leq 0.05) ; n s=$ valor de $\mathrm{F}$, no significativo $(p \geq 0.05)$.

Diversos autores coinciden en señalar que se logra mejor aclimatación de las plantas obtenidas in vitro cuando se establecen en sustratos que sean una mezcla de materia orgánica y materiales inertes que mejoren la porosidad para aireación y drenaje, en algunos casos también señalan la adición de nutrimentos por otros medios como soluciones nutritivas o fertilizaciones foliares que hacen disponibles los minerales en formas iónicas que la planta puede aprovechar en el corto plazo. Por ejemplo, Vilchez et al. (2007) encontraron que vitroplantas de sábila (Aloe vera L.) mostraron incrementos en número de hojas y altura cuando se establecieron en el sustrato humus de lombriz, además el crecimiento de las plantas fue más rápido y vigoroso, en comparación con plantas que se establecieron en arena de río.

Florio y Mogollón (2011), reportaron que grupos de vitroplantas de plátano Musa sp. cv. Roatán que se establecieron en un sustrato compuesto de $85 \%$ de turba v/v y $15 \%$ de vermiculita v/v, 90\% de plantas sobrevivió después de 30 días de aclimatación, y tuvieron $12.24 \mathrm{~cm}$ de altura, $13.87 \mathrm{~mm}$ de diámetro del brote, 6.38 hojas, $13.65 \mathrm{~cm}$ de longitud máxima de raíces, 1.7 y $0.64 \mathrm{~g}$ de biomasa fresca; 0.27 y $0.07 \mathrm{~g}$ de biomasa seca de los brotes y raíces, magnitudes superiores en comparación con plantas que estuvieron en otros sustratos: 1) aserrín de coco (100\%); 2) arena, aserrín de coco y cáscara de arroz $(1: 2: 1 \mathrm{v} / \mathrm{v}) ; 3)$ arena, aserrín de coco y cáscara de arroz y vermicompost (1:5:2:2 $\mathrm{v} / \mathrm{v})$. Los sustratos anteriores, ya sea que contengan materia orgánica o solo materiales inertes, deben tener porosidad adecuada para la aireación y drenaje. Así también, el abastecimiento de nutrimentos es benéfico para que las plantas logren mayor crecimiento y rendimiento. Abreu et al. (2007), al micropropagar $A$. fourcroydes trasfirieron las plantas a contenedores de poliespuma de 47 x $69 \mathrm{~cm}$ con 247 alvéolos cada uno con $30 \mathrm{~cm}^{3}$ de volumen, con sustrato de pulpa de henequén fermentado y zeolita en diferentes proporciones. 
Las plantas se establecieron en un invernadero que permitía el paso de $30 \%$ de la luz $\approx 558.74 \mathrm{y}$ $686.55 \mu \mathrm{mol} \mathrm{m} \mathrm{m}^{-2} \mathrm{~s}^{-1}$ ), éstas condiciones fueron generadas mediante el empleo de capas de malla sarán de color negro. Trascurridos 30 días, las plantas que se establecieron en el sustrato compuesto de $4.46 \mathrm{~kg}$ de pulpa de henequén y $5.54 \mathrm{~kg}$ de zeolita, $92 \%$ de las plantas sobrevivió y tuvieron en promedio $7.64 \mathrm{~cm}^{2}$ de área foliar y $59.43 \mathrm{mg}$ de peso seco total.

Por otra parte, Crespo-González et al. (2013) trabajaron con plantas micropropagadas in vitro de Agave tequilana Weber var. azul con una altura de $15-22 \mathrm{~cm}$ y 16 meses de edad, en donde evaluaron la respuesta de las plantas a los tratamientos establecidos a base de formulaciones de los sustratos orgánicos (polvo de coco (PC), turba (T) y composta de bagazo de agave (C), mezclados en una relación de volumen (v/v), considerándose los siguientes: 1) testigo: 80\% PC $+10 \% \mathrm{~T}+10 \% \mathrm{C}$; 2) $100 \% \mathrm{PC}$; 3) $100 \% \mathrm{~T}$; 4) $70 \% \mathrm{PC}+30 \% \mathrm{C}$; 5) $50 \% \mathrm{PC}+50 \% \mathrm{C}$; 6) $30 \%$ $\mathrm{PC}+70 \% \mathrm{C}$; y 7) $100 \% \mathrm{C}$. Además, las plantas de todos los tratamientos durante nueve meses recibieron entre 7 y 14 fertirriegos por mes con quelatos de hierro y manganeso, sulfato de magnesio, nitrato de calcio, y fórmulas N-P-K 18-18-18 (período septiembre-diciembre) y 1530-15 (período enero-mayo).

Las plantas que estuvieron en sustratos con composta al 30 y $50 \%$ desarrollaron la piña con diámetros de 4.7 y $5.07 \mathrm{~cm}$ y el tallo de 3.5 y $3.75 \mathrm{~cm}$, tuvieron 15 y 15.3 hojas, la hoja mayor fue de 55.4 y $51.3 \mathrm{~cm}$ de longitud, así como 3.6 y $4 \mathrm{~cm}$ de ancho, respectivamente, magnitudes que fueron superiores a los $3.63 \mathrm{~cm}$ de diámetro de piñas, $2.1 \mathrm{~cm}$ de diámetro de tallo, 9.8 hojas, con la hoja mayor de $36.9 \mathrm{~cm}$ de longitud y $3 \mathrm{~cm}$ de anchura, que tuvieron las plantas en el sustrato de $100 \%$ turba. Los autores concluyen que se obtienen plantas de mayor tamaño con los sustratos que contienen mayor cantidad de materia orgánica mezclada con otro material que proporcione buena aireación y filtración.

\section{Índice estomático en hojas adultas y jóvenes de plantas micropropagadas de Agave americana var. Oaxacensis al finalizar su aclimatización}

Las plantas micropropagadas que son trasferidas a condición ex vitro forman estructuras vegetativas durante su aclimatización en invernadero, donde la planta desarrolla hojas que muestran características diferentes respecto a las hojas formadas en condición in vitro: cutícula, parénquima empalizada y mesófilo. Entre los cambios observados, se menciona que las hojas nuevas formadas en condiciones de invernadero tienen cantidad diferente de estomas (Pospíšilová et al., 1999).

En la presente investigación se observó que, al inicio de su aclimatización, las hojas de las plantas de A. americana var. Oaxacensis micropropagadas tuvieron un índice estomático con promedio de $4.55 \%$ para la sección basal, $7 \%$ para la sección media y $7.93 \%$ para la sección distal, en cambio en el envés de las hojas se obtuvo un promedio de 4.16, 7.26 y 9.32\% para la sección basal, media y distal respectivamente. Al concluir esta etapa, el índice estomático se había incrementado en las distintas secciones de la hoja. En la superficie adaxial (haz) en su sección basal, media y distal, se tuvo en promedio 4.82, 9.06 y $10.67 \%$ de índice estomático, respectivamente, mientras que para la superficie abaxial (envés) se obtuvieron promedios de $5.93,9.24$ y $10.86 \%$ respectivamente. 
Cuadro 3. Efecto del sustrato y la fertilización sobre el índice estomático del haz y envés de hojas adultas de $A$. americana var. Oaxacensis después de 270 días de adaptación.

\begin{tabular}{lccccccc}
\hline \multicolumn{1}{c}{ Sust } & Fert $(\%)$ & $\begin{array}{c}\text { IEHB } \\
(\%)\end{array}$ & $\begin{array}{c}\text { IEHM } \\
(\%)\end{array}$ & IEHD $(\%)$ & $\begin{array}{c}\text { IEEB } \\
(\%)\end{array}$ & $\begin{array}{c}\text { IEEM } \\
(\%)\end{array}$ & IEED $(\%)$ \\
\hline $33.3 \% \mathrm{~T}: 66.6 \% \mathrm{~A}$ & 0 & $5.3 \mathrm{a}$ & $9.5 \mathrm{ab}$ & $12.6 \mathrm{a}$ & $6 \mathrm{a}$ & $9 \mathrm{a}$ & $11.6 \mathrm{ab}$ \\
& 50 & $5.2 \mathrm{a}$ & $8.9 \mathrm{ab}$ & $10.9 \mathrm{abc}$ & $6.7 \mathrm{a}$ & $9.8 \mathrm{a}$ & $11.1 \mathrm{ab}$ \\
& 100 & $4.5 \mathrm{a}$ & $9.5 \mathrm{ab}$ & $10.7 \mathrm{abc}$ & $6.6 \mathrm{a}$ & $9.6 \mathrm{a}$ & $9.7 \mathrm{~b}$ \\
& 0 & $5 \mathrm{a}$ & $7.5 \mathrm{~b}$ & $10.2 \mathrm{abc}$ & $5.3 \mathrm{a}$ & $8.3 \mathrm{a}$ & $9.6 \mathrm{~b}$ \\
& 50 & $4.6 \mathrm{a}$ & $9.5 \mathrm{ab}$ & $9.4 \mathrm{bc}$ & $6 \mathrm{a}$ & $10.7 \mathrm{a}$ & $12.2 \mathrm{a}$ \\
$75 \% \mathrm{~T}: 25 \% \mathrm{~A}: 33.3 \% \mathrm{~A}$ & 100 & $4.3 \mathrm{a}$ & $7.6 \mathrm{~b}$ & $8.9 \mathrm{c}$ & $5.2 \mathrm{a}$ & $8.4 \mathrm{a}$ & $10.4 \mathrm{ab}$ \\
& 0 & $4.6 \mathrm{a}$ & $9.5 \mathrm{ab}$ & $10.2 \mathrm{abc}$ & $5.3 \mathrm{a}$ & $9.2 \mathrm{a}$ & $11.6 \mathrm{ab}$ \\
& 50 & $4.9 \mathrm{a}$ & $10.1 \mathrm{a}$ & $11.8 \mathrm{ab}$ & $6 \mathrm{a}$ & $9.8 \mathrm{a}$ & $11.3 \mathrm{ab}$ \\
Sustrato & 100 & $4.6 \mathrm{a}$ & $9 \mathrm{ab}$ & $10.9 \mathrm{abc}$ & $5.8 \mathrm{a}$ & $7.9 \mathrm{a}$ & $9.8 \mathrm{~b}$ \\
Fertilización & & $\mathrm{ns}$ & $* *$ & $* *$ & $\mathrm{~ns}$ & $\mathrm{~ns}$ & $\mathrm{~ns}$ \\
Sust*Fert & $\mathrm{ns}$ & $\mathrm{ns}$ & $\mathrm{ns}$ & $\mathrm{ns}$ & $* *$ & $* *$ \\
\hline
\end{tabular}

Sust $=$ sustrato; Fert $=$ fertilización; $33.3 \% \mathrm{~T}: 66.6 \% \mathrm{~A}=$ una porción de turba combinada con dos de arena; $66.6 \% \mathrm{~T}$ : $33.3 \% \mathrm{~A}=$ dos porciones de turba combinada con una de arena; $75 \% \mathrm{~T}: 25 \% \mathrm{~A}=$ tres porciones de turba combinada con una de arena; IEHB= índice estomático haz sección base; IEHM= índice estomático haz sección media; IEHD= índice estomático haz sección distal; IEEB= índice estomático envés sección base; IEEM= índice estomático envés sección media; IEED $=$ índice estomático envés sección distal; ${ }^{* *}=$ valor de $\mathrm{F}$ significativo $(p \leq 0.01) ;{ }^{*}=$ valor de $\mathrm{F}$ significativo $(p \leq 0.05)$; ns= valor de F no significativo $(p \geq 0.05)$.

De acuerdo con el análisis de varianza, los niveles del factor sustrato tuvieron efectos significativos diferentes $(p<0.01)$ sobre la variable índice estomático del haz en la sección media y distal de la hoja de tamaño mayor de Agave americana var. Oaxacensis (Cuadro 3). Los niveles del factor tipo de riego mostraron efectos significativos $(p<0.01)$ diferentes únicamente para las variables índice estomático en envés sección media y distal. La interacción sustrato-tipo de riego tuvo efecto significativo, únicamente para la variable índice estomático en el envés sección distal de la hoja.

En lo que respecta al índice estomático, determinado en las hojas nuevas formadas ex vitro, se observó que la distribución de los estomas es más homogénea que en condiciones in vitro ya que la sección media y apical tanto del haz como del envés presentan valores de índice estomático muy similares (Cuadro 4). Las hojas que las plantas de A. americana var. Oaxacensis formaron in vitro presentaron menor índice estomático, respecto de las hojas nuevas que la planta desarrolló durante su adaptación.

Esta característica podría deberse a que hubo un aumento del tamaño de las células epidérmicas (Figura 1); por lo tanto, en el campo de observación del microscopio se observaron menor cantidad de ellas, con lo cual se vio afectado el denominador de la fórmula para calcular el índice estomático. Stranburger et al. (1986), mencionan que el número de células epidérmicas grandes que reduce el denominador de la fórmula de IE por unidad de superficie analizada posiblemente está relacionado con la optimización de la capacidad para el almacenamiento de agua. 

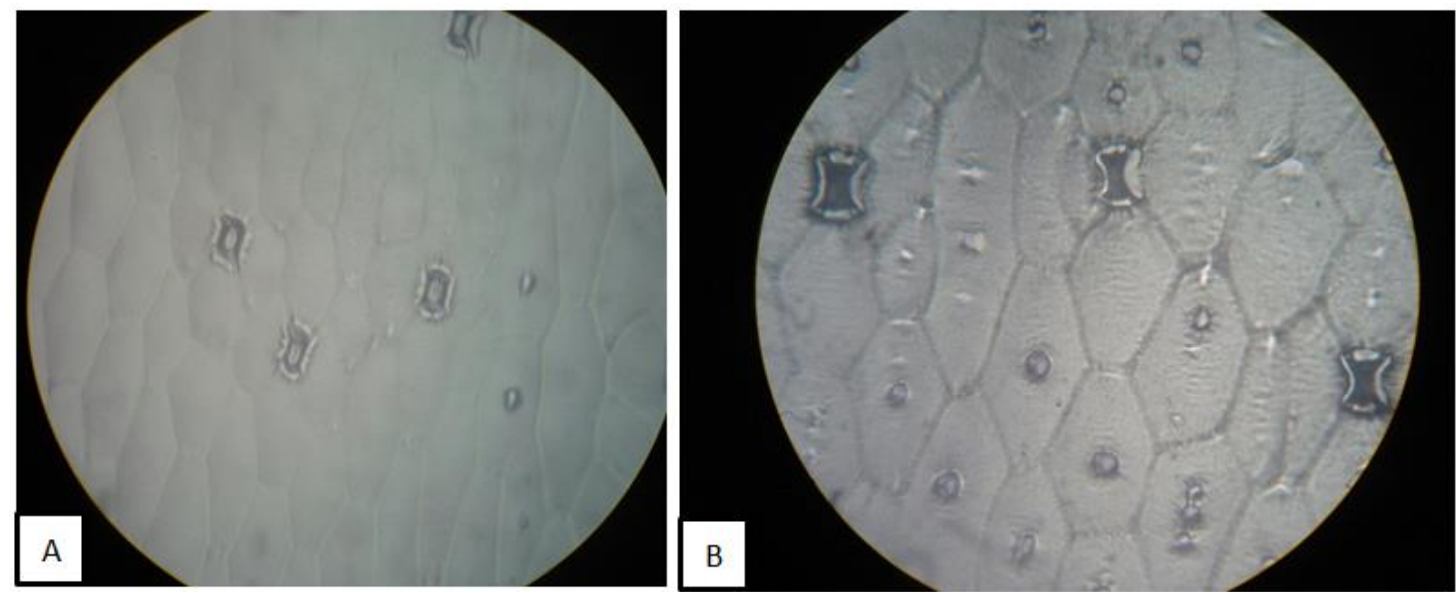

Figura 1. Estomas observados en el microscopio en un campo de 40x. (A) estomas presentes en $A$. americana var. Oaxacensis en cultivo in vitro; y (B) estomas en plantas adaptadas en invernadero.

Los análisis de varianza mostraron que el factor sustrato, el factor tipo de riego y la interacción sustrato-tipo de riego presentaron efectos significativos sobre IEEM, pero no sobre las variables IEHB, IEHM, IEHD, IEEB y IEED. Lo anterior, denota que los valores de índice estomático determinados en las diferentes secciones de las hojas nuevas de A. americana var. Oaxacensis, no se ve influido ni por el tipo de sustrato ni por el suministro de nutrimentos en el riego (Cuadro 4).

Cuadro 4. Efecto del sustrato y abastecimiento de nutrimentos en el riego sobre el índice estomático del haz y envés de hojas nuevas de $A$. americana var. oaxacencis después de 270 días de adaptación.

\begin{tabular}{|c|c|c|c|c|c|c|c|}
\hline Sust & Fert $(\%)$ & $\begin{array}{c}\text { IEHB } \\
(\%)\end{array}$ & $\begin{array}{c}\text { IEHM } \\
(\%)\end{array}$ & $\begin{array}{c}\text { IEHD } \\
(\%)\end{array}$ & $\begin{array}{c}\text { IEEB } \\
(\%)\end{array}$ & IEEM (\%) & $\begin{array}{l}\text { IEED } \\
(\%)\end{array}$ \\
\hline \multirow[t]{3}{*}{$33.3 \% \mathrm{~T}: 66.6 \% \mathrm{~A}$} & 0 & $9.2 \mathrm{a}$ & $12.1 \mathrm{a}$ & 13.5 & $8.7 \mathrm{a}$ & $13.8 \mathrm{a}$ & $14.6 \mathrm{a}$ \\
\hline & 50 & $8.6 \mathrm{a}$ & $12.3 \mathrm{a}$ & 12 & $9.4 \mathrm{a}$ & $11.9 \mathrm{abc}$ & $12.9 \mathrm{a}$ \\
\hline & 100 & $7.5 \mathrm{a}$ & $11.2 \mathrm{a}$ & 11.6 & $8.3 \mathrm{a}$ & $10.5 \mathrm{bc}$ & $12 \mathrm{a}$ \\
\hline \multirow[t]{3}{*}{$66.6 \% \mathrm{~T}: 33.3 \% \mathrm{~A}$} & 0 & $6.5 \mathrm{a}$ & $10.4 \mathrm{a}$ & 11.5 ; & $9.1 \mathrm{a}$ & $10.3 \mathrm{bc}$ & $12 \mathrm{a}$ \\
\hline & 50 & $8.2 \mathrm{a}$ & $12.1 \mathrm{a}$ & 11.7 a & $8.1 \mathrm{a}$ & $12.3 \mathrm{ab}$ & $12.6 \mathrm{a}$ \\
\hline & 100 & $8.7 \mathrm{a}$ & $10.4 \mathrm{a}$ & 11.4 a & $9.2 \mathrm{a}$ & $12.1 \mathrm{abc}$ & $11.9 \mathrm{a}$ \\
\hline \multirow[t]{3}{*}{$75 \% \mathrm{~T}: 25 \% \mathrm{~A}$} & 0 & $7.5 \mathrm{a}$ & $10.5 \mathrm{a}$ & 10.9 a & $9.1 \mathrm{a}$ & $11.4 \mathrm{bc}$ & $12.5 \mathrm{a}$ \\
\hline & 50 & $7.6 \mathrm{a}$ & $10.9 \mathrm{a}$ & 11.7 a & $8.8 \mathrm{a}$ & $11.4 \mathrm{bc}$ & $13.1 \mathrm{a}$ \\
\hline & 100 & $7.6 \mathrm{a}$ & $9.7 \mathrm{a}$ & 10.5 & $7.6 \mathrm{a}$ & $9.9 \mathrm{c}$ & $12.1 \mathrm{a}$ \\
\hline Sustrato & & ns & ns & ns & ns & $*$ & ns \\
\hline Fertilización & & $\mathrm{ns}$ & ns & ns & ns & $*$ & $\mathrm{~ns}$ \\
\hline Sust $*$ Fert & & ns & $\mathrm{ns}$ & $\mathrm{ns}$ & $\mathrm{ns}$ & $* *$ & $\mathrm{~ns}$ \\
\hline
\end{tabular}

Sust= sustrato; Fert= fertilización; 33.3\% $\mathrm{T}: 66.6 \% \mathrm{~A}=$ una porción de turba combinada con dos de arena; $66.6 \% \mathrm{~T}$ : $33.3 \% \mathrm{~A}=$ dos porciones de turba combinada con una de arena; $75 \% \mathrm{~T}: 25 \% \mathrm{~A}=$ tres porciones de turba combinada con una de arena; $\mathrm{IEHB}=$ índice estomático haz sección base; IEHM= índice estomático haz sección media; IEHD= índice estomático haz sección distal; IEEB= índice estomático envés sección base; IEEM= índice estomático envés sección media; IEED= índice estomático envés sección distal; ${ }^{* *}=$ valor de F significativo $(p<0.01) ;{ }^{*}=$ valor de F significativo $(p \leq 0.05) ; \mathrm{ns}=$ valor de F no significativo $(p>0.05)$. 
Los resultados del índice estomático mayor en hojas jóvenes de A. americana var. Oaxacensis aclimatadas, coinciden con Bazaldua-Muñoz et al. (2008), quienes reportan que plantas de Physalis ixocarpa micropropagadas presentaron un incremento gradual de densidad estomatica tanto del haz como del envés en función del tiempo de aclimatación.

Contenido de clorofila en hojas adultas y jóvenes de plantas micropropagadas de $A$. americana var. Oaxacensis adaptadas en invernadero

De acuerdo con el análisis de varianza (Cuadro 5) se encontraron diferencias significativas $(p \leq$ 0.001) sobre el contenido de clorofila en hojas adultas y jóvenes de A. americana var. Oaxacensis en respuesta al sustrato en el que se establecieron y al abastecimiento de nutrimentos en el riego, que la interacción sustrato-tipo de riego no tuvo efecto significativo. Las plantas de agave micropropagadas que estuvieron en los sustratos con dos y tres porciones de turba combinados con una de arena y además recibieron solución nutritiva al 100\% respectivamente, sus hojas de mayor edad tuvieron de 43.64 y $42.91 \mu \mathrm{g} \mathrm{cm}^{-2}$ de clorofila, magnitudes significativamente (Tukey, $p<$ 0.05) mayores a los $23.95 \mu \mathrm{g} \mathrm{cm}^{-2}$ que tuvieron las hojas de las plantas que se establecieron en el sustrato con una porción de turba combinado con dos de arena y que no recibieron solución nutritiva adicional (Cuadro 5). Las hojas jóvenes de las plantas de A. americana var. Oaxacensis tuvieron más clorofila que las hojas de mayor edad. El valor más alto de clorofila $75.85 \mu \mathrm{g} \mathrm{cm}^{-2} \mathrm{se}$ observó en las hojas jóvenes de las plantas que estuvieron en el sustrato con dos porciones de turba combinado con una de arena y que además se fertirrigaron con la solución Steiner al 100\%.

Cuadro 5. Efecto del sustrato y abastecimiento de nutrimentos en el riego sobre el contenido de clorofila en hojas de $A$. americana var. Oaxacensis después de 270 de su adaptación en invernadero.

\begin{tabular}{lccc}
\hline \multirow{2}{*}{ Sustrato } & Fertilización $(\%)$ & \multicolumn{2}{c}{ Clorofila $\left(\mu \mathrm{g} \mathrm{cm}^{-2}\right)$} \\
\cline { 2 - 4 } $33.3 \% \mathrm{~T}:$ 66.6\%A & 0 & $23.95 \mathrm{~d}$ & $38.9 \mathrm{~d}$ \\
& 50 & $26.78 \mathrm{~cd}$ & $46.57 \mathrm{dc}$ \\
& 100 & $30.27 \mathrm{bcd}$ & $50.98 \mathrm{bcd}$ \\
66.6\%T: 33.3\%A & 0 & $32.92 \mathrm{bc}$ & $55.75 \mathrm{bcd}$ \\
& 50 & $35.68 \mathrm{abc}$ & $64.14 \mathrm{abc}$ \\
& 100 & $43.64 \mathrm{a}$ & $75.85 \mathrm{a}$ \\
$75 \% \mathrm{~T}: 25 \% \mathrm{~A}$ & 0 & $35.68 \mathrm{ab}$ & $54.67 \mathrm{bcd}$ \\
& 50 & $38.69 \mathrm{ab}$ & $61.09 \mathrm{abc}$ \\
Sustrato & 100 & $42.91 \mathrm{a}$ & $67.11 \mathrm{ab}$ \\
Fertilización & & $* *$ & $* *$ \\
Sustrato*fertilización & & ns & $\mathrm{ns}$ \\
\hline
\end{tabular}

${ }^{* *}=$ valor de F significativo $(p \leq 0.01) ;{ }^{*}=$ valor de F significativo $(p<0.05)$; ns $=$ valor de $\mathrm{F}$ no significativo $(p \geq 0.05)$. Medias con letras iguales no son estadísticamente diferentes (Tukey, $p<0.05$ ); 33.3\% T: 66.6\% A = una porción de turba combinada con dos de arena; $66.6 \% \mathrm{~T}: 33.3 \% \mathrm{~A}=$ dos porciones de turba combinada con una de arena; $75 \% \mathrm{~T}: 25 \% \mathrm{~A}=$ tres porciones de turba combinada con una de arena. 
El mayor crecimiento de las plantas establecidas en estos sustratos se podría atribuir a la disponibilidad de una mayor cantidad de nutrientes en el sustrato con proporción de turba alta y los aportados por la solución nutritiva. Lo anterior concuerda con Wu et al. (2008), quienes reportan que el estado nutricional de las plantas tiene relación positiva con el contenido de clorofila y a su vez con la actividad fotosintética. Mientras que Zarco-Tejada et al. (2004), mencionan que en plantas con crecimiento vigoroso generalmente se espera que contengan concentraciones altas de clorofila, en relación con plantas menos vigorosas.

\section{Conclusiones}

El sustrato y el abastecimiento nutrimental durante la aclimatización de plantas de A. americana var. Oaxacensis micropropagadas determinan su crecimiento y desarrollo. Todos los tratamientos probados permitieron que el total de plantas sobrevivieran, sin embargo, las que se establecieron en sustratos con dos porciones de turba combinado con una de arena y que además recibieron solución nutritiva al 100\% de concentración, mostraron características mejores de crecimiento y sus hojas adultas y jóvenes tuvieron los contenidos más altos de clorofila. Los valores de índice estomático en hojas adultas y jóvenes de las plantas no se vieron afectados ni por el tipo de sustrato ni la fertilización.

Durante la aclimatización de A. americana var. Oaxacensis se recomienda utilizar sustratos que contengan suficiente materia orgánica, con buen drenaje y aireación, además de considerar la fertilización líquida como una forma rápida de hacer disponibles los nutrimentos.

\section{Literatura citada}

Abreu, E.; González, G.; Ortiz, R.; Rodríguez, P.; Domech, R. y Garriga, M. 2007. Evaluación de vitroplantas de henequén (Agave fourcroydes Lem) durante la fase de aclimatización. Cultivos Tropicales. 28(1):5-11.

Arizaga, S. and Ezcurra, E. 1995. Insurance against reproductive failure in a semelparous plant: bulbil formation in Agave macroacantha flowering stalks. Oecologia. 101(3):329-334. doi: 10.1007/BF00328819.

Arzate-Fernández, A. M.; Piña-Escutia, J. L.; Norman-Mondragón, T. H.; Reyes-Díaz, J. I.; Guevara-Suárez, K. L. y Vázquez-García, L. M. 2016. Regeneración de agave mezcalero (Agave angustifolia Haw.) a partir de embriones somáticos encapsulados. Rev. Fitotec. Mex. 39(4):359-366.

Aureoles-Rodríguez, F.; Rodríguez-de la O, J. L.; Legaria-Solano, J. P.; Sahagún-Castellanos, J. y Peña Ortega, M. G. 2008. Propagación in vitro del 'maguey bruto' (Agave inaequidens Koch), una especie amenazada de interés económico. Revista Chapingo Ser. Hortic. 14(3):263-269.

Bazaldua-Muñoz, C.; Ventura-Zapata, E.; Salcedo-Morales.; Maldonado-Amaya, U. y LópezGarcía, A. 2008. Densidad estomatal y potencial hídrico en plantas de tomate (Physalis ixocarpa Brot.), propagadas por cultivo de meristemos. Revista Chapingo Ser Hortic. 14(2):147-150.

Carneiro-dos Santos, F.; de Olivera, D, Q. S. R.; Rodríguez, P. A.; Neves, N. M. y Souza, S. K. 2014. Embriogénesis somática en Agave Sisalana Perrine: inducción, caracterización anatómica y regeneración. Pesquisa Agropecuária Tropical. 44(3):294-303. 
CONABIO. 2006. Comisión Nacional para el Conocimiento y Uso de la Biodiversidad. Mapa de mezcales y diversidad. https://www.biodiversidad.gob.mx/usos/mezcales/mMapa.html.

Coste, S.; Baraloto, C.; Leroy, C.; Marcon, E.; Renaud, A.; Richardson, A. D.; Roggy, J. C.; Schimann, H.; Uddling, J. and Herault, B. 2010. Assessing foliar chlorophyll contents with the SPAD-502 chlorophyll meter: a calibration test with thirteen tree species of tropical rainforest in French Guiana. Annual Forest Sci. 1(67):1-5. doi: 10.1051/forest/2010020

Crespo-González, M. R.; González, E. D. R.; Rodríguez, M. R.; Rendón, S. L. A.; del Real, L. J. I. y Torres, M. J. P. 2013. Evaluación de la composta de bagazo de agave como componente se sustratos para producir plántulas de agave azul tequilero. Rev. Mex. Cienc. Agríc. 4(8):1161-1173.

Cruz-García, H.; Campos-Ángeles, G. V.; Enríquez-del Valle, J. R.; Velasco-Velasco, V. A y Rodríguez-Ortiz, G. 2017. Senescencia foliar en plantas micropropagadas de Agave americana durante su aclimatización. Rev. Mex. Cienc. Agríc. 8(2):381-391.

Domínguez, R. M. S.; González, M. L.; Rosales, C.; Quiñones, C.; Delgadillo, L.; Mireles, J. y Pérez, B. E. 2008. El cultivo in vitro como herramienta para el aprovechamiento, mejoramiento y conservación de especies del género Agave. Investigación y Ciencia. 16(41):53-62.

Enríquez-del Valle, J. R. 2008. La propagación y crecimiento de agaves. Fundación Produce Oaxaca, A.C. Instituto Tecnológico del Valle de Oaxaca. Oaxaca. México. 46. p.

Enríquez-del Valle, J. R.; Alcara, V. S. E.; Rodríguez-Ortiz, G.; Miguel, L. M. E. y Manuel, V. C. 2016. Fertirriego en vivero a plantas de Agave potatorum Zucc micropropagadasaclimatizadas. Rev. Mex. Cienc. Agríc. 7(5):1167-1177.

Florio, S. y Mogollón, N. 2011. Efecto del tipo de sustrato en la aclimatización de vitroplantas de plátano Hartón Gigante (Musa AAB). Rev. Fac. Agron. 28(1):99-109.

García-Mendoza, A. J. 2007. Los agaves de México. Ciencias. 87(jul-sep):14-23.

García-Mendoza, A. J. 2011. Flora del Valle de Tehuacán-Cuicatlán. Agavaceae. Fascículo 88. Jardín Botánico. Instituto de Biología-Universidad Nacional Autónoma de México (UNAM). 95 p.

Garriga, C. M.; González, O. G.; Alemán, G. S.; Abreu, C. E.; Quiroz, B. K.; Caligari, P. D. S. and García, G. R. 2010. Management of auxin-cytokinin interactions to improve micropropagation protocol of henequen (Agave fourcroydes Lem.). Chilean J. Agric. Res. 70(4):545-551.

George, E. F. and Debergh, P. C. 2008. Micropropagation: uses and methods. In: George, E. F.; Hall, M. and De Klerk, G. (Eds.). Plant propagation by tissue culture. $3^{\text {th }}$ (Ed.). The Bacground. Springer. 29-64 pp.

González, M.; Mogollón, N.; Alvarado, G.; Giménez, A. y Capote, T. 2012. Efecto del medio de cultivo in vitro y la fuente nitrogenada sobre el crecimiento del cocuy (Agave cocui Trelease). Bioagro. 24(1):39-44.

Mantovani, A. 1999. A Method to improve leaf succulence quantification. Brazilian Archives of Biology and Technology. 42(1):1-6. http://dx.doi.org/10.1590/S1516-89131999000 100002.

Moran, R. 1982. Formulae for determination of chlorophyllous pigments estracted with N, NDimethylformamide. Plant Physiol. 69(6):1376-1381. doi: https://doi.org/10.1104/pp.69.6.1376.

Nobel, P. S. 1998. Los incomparables agaves y cactus. Editorial Trillas. México. 211 p.

Palma-Cruz, F. de J. 2000. Agaves productores de fibras duras en el estado de Oaxaca, México. Boletín de la Sociedad Botánica de México. 66(1):93-102. DOI: 10.17129/botsci.1615. 
Pospíšilová, J.; Tichá, I.; Kadleček, P.; Haisel, D. and Plzáková, Š. 1999. Acclimatization of micropropagated plants to ex vitro conditions. Biology Plantarum. 42(4):481-497. doi: 10.1023/A:1002688208758.

Santacruz-Ruvalcaba, F.; Torres, M. I. y Portillo, L. 2008. Micropropagacion de Agave tequilana Weber cv. Azul; Problemas y perspectivas. Scientia-CUCBA. 10(1-2): 7-20.

Santíz, J. A.; Rincón-Rosales, R y Gutiérrez-Miceli, F. A. 2012. Propagación in vitro de Agave grijalvensis $\mathrm{B}$. Ullrich, una especie endémica de Chiapas bajo protección especial. Gayana Botánica. 69(especial):23-30.

Steiner, R. 1984. The universal nutrient solution. In: proceedings of IWOSC $19846^{\text {th }}$ International Congress on Soilless Culture. April 29-May 5. Wageningen, The Netherlands. 633-650 pp.

Stranburger, E.; Noll, F.; Schenck, H. y Schimper, A. 1986. Tratado de botánica. EM Marin. Barcelona, España. 1098 p.

Vilchez, J.; Ramírez, E.; Villasmil, M.; Albany, N.; León de Sierralta, S. y Molina, M. 2007. Aclimatación de vitroplantas de zabila (Aloe vera (L.) Burm. F): efectos del sustrato. Rev. Fac. Agron. 24(1):57-61.

Wilkinson, H. 1979. The plant surface (mainly leaf). In: C. R. Metcalfe y Chalk (Eds.). Anatomy of dicotiledons. Oxford Claredous Press. London. 97-165 pp.

Wu, C.; Niu, Z.; Tang, Q. and Wang, W. 2008. Estimating chlorophyll content from hyperspectral vegetation indices: Modeling and validation. Agricultural and Forest Meteorology. 148(89):1230-1241. doi:10.1016/j.agrformet.2008.03.005.

Zarco-Tejada, P.; Miller, J.; Harron, J.; Hu, B.; Noland, T.; Goel, N.; Mohammed, G. and Sampson, P. 2004. Needle chlorophyll content estimation through model inversion using hyperspectral data from boreal conifer forest canopies. Remote Sensing of Environment. 89(2):189-199. doi:10.1016/j.rse.2002.06.002. 